\title{
Clinical characteristics of brain metastases from lung cancer according to histological type: Pretreatment evaluation and survival following whole-brain radiotherapy
}

\author{
TETSUYA KOMATSU, ETSUO KUNIEDA, YUKIO OIZUMI, YOSHIFUMI TAMAI and TAKESHI AKIBA \\ Department of Radiation Oncology, Tokai University School of Medicine, Isehara, Kanagawa 259-1193, Japan
}

Received December 22, 2012; Accepted April 30, 2013

DOI: $10.3892 / \mathrm{mco} .2013 .130$

\begin{abstract}
The histological type of lung cancer in patients with brain metastases may affect response to treatment and survival. We evaluated the clinical characteristics of brain metastases from lung cancer according to histological type in 70 consecutive patients with brain metastases from histologically confirmed lung cancer, who had been previously treated with whole-brain radiotherapy (WBRT). Histological type was divided into three categories: adenocarcinoma, small-cell lung carcinoma (SCLC) and other non-small cell lung cancer (NSCLC). The number, size and maximum diameter of brain metastases, the size and maximum diameter of peritumoral edema, the ratio of tumor and peritumoral edema, the asymptomatic ratio, the tumor size reduction rate, the complete response (CR) rate, the intracranial progression-free survival (PFS) and the overall survival (OS) were also evaluated. The median survival time for all patients was 26.2 weeks. Patients with SCLC exhibited a significantly smaller edema size and maximum diameter of edema compared to patients with other NSCLC $(\mathrm{P}=0.016$ and 0.010 , respectively). The ratio of tumor and peritumoral edema was also significantly lower in patients with SCLC compared to that in patients with adenocarcinoma and other NSCLC $(\mathrm{P}=0.001)$. Significant differences in intracranial PFS and OS between adenocarcinoma and other NSCLC were also observed $(\mathrm{P}=0.018$ and 0.004 , respectively). Patients with adenocarcinoma who were treated with epidermal growth factor receptor (EGFR) tyrosine kinase inhibitors (TKIs) following WBRT, demonstrated a significant improvement in intracranial PFS and OS $(\mathrm{P}=0.008$ and 0.004 , respectively). The findings presented in this study may provide useful information for the management of brain metastases. Patients with SCLC exhibit a tendency to develop peritumoral edema to a lesser extent, compared to patients with other histological tumor types. Findings in the present study suggest that
\end{abstract}

Correspondence to: Dr Tetsuya Komatsu, Department of Radiation Oncology, Tokai University School of Medicine, 143 Shimokasuya, Isehara, Kanagawa 259-1193, Japan

E-mail: komat@tokai-u.jp

Key words: tyrosine kinase inhibitors, peritumoral edema patients with adenocarcinoma, particularly those treated with EGFR-TKIs, exhibit improved survival rates.

\section{Introduction}

Many cancer patients develop brain metastases, which lead to significant morbidity and decreased survival. Although no significant difference in survival has been reported in patients with different primary lesions (1), a previous large retrospective study revealed that the prognosis following the development of brain metastases was associated with the type of the primary lesion (2). The American Society for Radiation Oncology guidelines recommended that the results of randomized trials be interpreted with caution, due to the inclusion of patients with different primary cancers (3). However, lung cancer is the most common primary tumor accounting for more than half of all cases of brain metastases $(2,4)$. Approximately $25 \%$ of patients with lung cancer develop brain metastases during the course of their illness $(5,6)$ and their prognosis is poor, with a median survival time (MST) of 3-5 months (4-6).

Treatment approaches for patients with brain metastases vary depending on the lesion number and size. Selected patients with a potentially favorable prognosis may be suitable for stereotactic radiosurgery (SRS) (3). However, for patients with a large tumor or those with $>3$ lesions, whole-brain radiotherapy (WBRT) alone remains the standard treatment. The histological type of lung cancer may affect response to treatment and survival. Newly developed drugs and epidermal growth factor receptor (EGFR) tyrosine kinase inhibitors (TKIs) have been shown to affect treatment outcome, depending on the histological tumor type (7-9). These findings suggested that the histological assessment of lesions in patients with brain metastases from lung cancer may be beneficial. However, the effect of histological type on survival remains controversial. Bergqvist et al noted that adenocarcinoma was associated with the most favorable prognosis (10). Similarly, Kepka et al reported that patients with adenocarcinoma had a better prognosis compared to those with squamous cell carcinoma (11). Conversely, other studies reported that the survival time following WBRT did not differ among different histological types (12-14).

Although the effect of histological type on survival following WBRT remains unclear, previous studies have indi- 
cated that the use of EGFR-TKIs for the treatment of brain metastases following WBRT may affect patient survival (15). The aim of this study was to assess the survival of patients with lung cancer, defined according to histological type, following WBRT. In addition, pretreatment clinical characteristics, particularly peritumoral edema, were evaluated according to histological type, since few studies have addressed this subject. This retrospective study assessed the pre- and post-treatment features of brain metastases from lung cancer according to histological type and may provide beneficial information for the management of brain metastases in lung cancer patients.

\section{Patients and methods}

Patients and classification. The present study was approved by the Institutional Review Board of our hospital (12R072). Between September, 2005 and April, 2011, a total of 102 patients underwent WBRT alone, excluding prophylactic cranial irradiation, for brain metastases from lung cancer at our institution. No patients underwent planned WBRT in combination with surgery or stereotactic radiosurgery (SRS). Out of the 102 patients, 94 patients completed the prescribed radiation dose. To evaluate the pretreatment characteristics by imaging, selection was limited to patients who underwent contrast-enhanced magnetic resonance imaging (MRI) within 2 weeks prior to the initiation of radiotherapy. Thirteen patients who had been diagnosed using computed tomography or non-contrast-enhanced MRI were excluded. Nine patients in whom the histological type of the primary tumor could not be determined and 2 patients with carcinomatous meningitis were also excluded. The remaining 70 patients underwent WBRT within 2 weeks after the diagnosis of brain metastases. WBRT was performed using lateral opposed fields with a 6-MV linear accelerator. Lesions were divided into 3 categories according to histopathological type: adenocarcinoma, small-cell lung carcinoma (SCLC) and other non-small cell lung cancer (NSCLC). Recursive partitioning analysis (RPA) as suggested by Gaspar et al (16) was evaluated in all 70 patients.

Assessment of pretreatment factors. To determine pretreatment clinical characteristics, the following factors were evaluated according to pathological type: number, size and maximum diameter of brain metastases; size and maximum diameter of peritumoral edema; ratio of tumor and peritumoral edema; and asymptomatic ratio. The asymptomatic ratio refers to the proportion of symptom-free patients among the total number of patients with brain metastases. Tumor size was measured as A $x \mathrm{~B}$, where $\mathrm{A}$ is the maximum diameter of the tumor $(\mathrm{mm})$, recognized as an enhanced lesion on the axial T1-weighted gadolinium-diethylenetriaminepentaacetic acid contrast-enhanced MRI and B is the maximum diameter ( $\mathrm{mm}$ ) perpendicular to A. Edema size was also measured in the same or a different plane across the tumor by using fluid-attenuated inversion recovery (FLAIR) imaging. The ratio of tumor and peritumoral edema (PE-index) was calculated using the equation:

$$
P E-\text { index }=\left\{\sum_{n=1}^{n}\left(\frac{\text { edema size } i}{\text { tumor number } \leq 5)}\right)\right\} / n
$$

If the number of metastases was $>5$, objects were limited to a maximum of 5 lesions in descending order from the largest tumor. Using this method, when peritumoral edema was almost absent, the PE-index was $\sim 1.0$.

Assessment of post-treatment factors. To determine post-treatment clinical characteristics, the following factors were evaluated according to histological type: tumor size reduction rate, complete response (CR) rate, intracranial progression-free survival (PFS) and overall survival (OS). Tumor size reduction rate was calculated by the following equation, using images in which the best response was observed: tumor size reduction rate $=($ sum of pretreatment MRI tumor size- sum of post-treatment MRI tumor size)/(sum of pretreatment MRI tumor size) x 100 .

The interval of follow-up MRI was 1-3 months and CR was defined as the status for which the sum of the tumor size was $<10 \mathrm{~mm}^{2}$. To assess the effect of EGFR-TKIs on survival, a comparison of intracranial PFS and OS between adenocarcinoma patients treated with and those not treated with EGFR-TKIs following WBRT was also performed. Gefitinib (Iressa; AstraZeneca, Macclesfield, UK) was used as an EGFR-TKI in 10 patients and erlotinib (Tarceva; OSI Pharmaceutics Inc., Melville, NY, USA) was used in 7 patients. A combination of gefitinib and erlotinib was used in 2 patients, at a daily dose of 250 and $150 \mathrm{mg}$, respectively. Intracranial PFS was measured from the initiation of radiotherapy to the date of progression of brain lesions on MRI or death from any cause. OS was measured from the start of radiotherapy to the date of death. Surviving patients were censored at the time of their last follow-up.

Statistical analysis. Statistical analyses were performed using the software package IBM SPSS Statistics 19. Proportions were compared using the Pearson's Chi-square test. Survival rates were calculated using the Kaplan-Meier method and compared using the log-rank test. The Kruskal-Wallis test was used for comparison among three categories for continuous variables. When significant differences were detected, the Mann-Whitney $U$ test was used to detect the difference between two categories.

\section{Results}

Patient characteristics and survival. Patient characteristics are provided in Table I. Adenocarcinoma was the most common pathological type, followed by SCLC and squamous cell carcinoma. Eight patients (11.4\%) were classified as recursive partitioning analysis (RPA) class I, 45 (64.3\%) were RPA class II and 17 (24.3\%) were RPA class III. Motor weakness was the most frequent symptom and brain metastases in asymptomatic patients were detected during the routine follow-up MRI. All patients were Japanese.

The median dose of WBRT was 30 Gy (range, 24-50 Gy). Dose fraction sizes were $2 \mathrm{~Gy}$ in 23 patients, $2.4 \mathrm{~Gy}$ in 3 patients, $2.5 \mathrm{~Gy}$ in 7 patients and $3 \mathrm{~Gy}$ in 37 patients. Five patients who presented with large metastases received an additional boost of irradiation limited to large lesions at a dose of $20 \mathrm{~Gy}$ in 10 fractions, 9 Gy in 3 fractions or 15 Gy in 5 fractions, depending on the preference of the physicians. 
Table I. Patient characteristics.

\begin{tabular}{|c|c|c|}
\hline Characteristics & No. of patients & $\%$ \\
\hline \multicolumn{3}{|l|}{ Age (years) } \\
\hline Median, 64 (range, 31-82) & 70 & 100 \\
\hline \multicolumn{3}{|l|}{ Gender } \\
\hline Male & 52 & 74.3 \\
\hline Female & 18 & 25.7 \\
\hline \multicolumn{3}{|l|}{ KPS } \\
\hline $100-80$ & 40 & 57.1 \\
\hline $70-50$ & 27 & 38.6 \\
\hline $40-10$ & 3 & 4.3 \\
\hline \multicolumn{3}{|l|}{ Histology } \\
\hline Adenocarcinoma & 44 & 62.9 \\
\hline Small-cell lung carcinoma & 15 & 21.4 \\
\hline Others & 11 & 15.7 \\
\hline Squamous cell carcinoma & 6 & \\
\hline LCNEC & 2 & \\
\hline Mucoepidermoid carcinoma & 1 & \\
\hline Pleomorphic carcinoma & 1 & \\
\hline Large-cell carcinoma & 1 & \\
\hline \multicolumn{3}{|l|}{ RPA class } \\
\hline $\mathrm{I}$ & 8 & 11.4 \\
\hline II & 45 & 64.3 \\
\hline III & 17 & 24.3 \\
\hline \multicolumn{3}{|l|}{ Symptom } \\
\hline Motor weakness & 8 & 11.4 \\
\hline Headache & 6 & 8.6 \\
\hline Speech difficulties & 6 & 8.6 \\
\hline Light-headedness & 6 & 8.6 \\
\hline Visual disturbance & 3 & 4.3 \\
\hline Vomiting & 3 & 4.3 \\
\hline Numbness & 2 & 2.9 \\
\hline Dizziness and vertigo & 2 & 2.9 \\
\hline Disorientation & 2 & 2.9 \\
\hline Seizure & 1 & 1.4 \\
\hline None & 31 & 44.3 \\
\hline
\end{tabular}

KPS, Karnofsky performance status; LCNEC, large-cell neuroendocrine carcinoma; RPA, recursive partitioning analysis.

Contrast-enhanced MRI scans following WBRT were available for evaluation for 50 patients who did not experience clinical deterioration. The median survival time (MST) for all patients was 26.2 weeks (95\% CI, 19-35 weeks), with a median follow-up time of 24 weeks. At the time of assessment, 7 of the 18 censored patients were alive.

Reirradiation for intracranial regrowth following WBRT was performed in 5 patients with adenocarcinoma (4 were treated with a cyberknife and 1 with a $\gamma$-knife) and in 4 patients with SCLC (3 were treated with WBRT and 1 with a $\gamma$-knife). In adenocarcinoma patients, the MST following the completion of reirradiation was 27 weeks (range, 4-34 weeks) and in
SCLC patients, the MST was 21 weeks (range, 8-114 weeks). No significant difference was observed between the two irradiated groups $(\mathrm{P}=0.711)$.

Clinical characteristics according to histological lesion type. Table II shows the clinical characteristics according to histological type. Total tumor size refers to the sum of each tumor size up to 5 lesions and total edema size refers to the sum of each edema size up to 5 lesions. The distribution of the number of brain metastases, tumor size and maximum tumor diameter did not significantly correlate with histological type. However, significant differences in edema size, maximum diameter of edema, PE-index and tumor size reduction rate were observed among the three histological categories $(\mathrm{P}=0.041,0.025$, $<0.001$ and $<0.001$, respectively).

Steroids were administered to improve symptoms caused by the metastases or the adverse effects of radiotherapy following evaluation of the lesions on MRI in 38 patients. The daily dose of dexamethasone was $2 \mathrm{mg}$ in 14 patients (9 with adenocarcinoma, 2 with SCLC and 3 with other NSCLC), $4 \mathrm{mg}$ in 17 patients (10 with adenocarcinoma, 3 with SCLC and 4 with other NSCLC), $6 \mathrm{mg}$ in 2 patients with adenocarcinoma and $8 \mathrm{mg}$ in 5 patients ( 3 with adenocarcinoma and 2 with SCLC). No significant differences were observed between these groups using the Chi-square test $(\mathrm{P}=0.797)$. Comparison of histological categories revealed differences in clinical characteristics based on histological type. Patients with SCLC exhibited a significantly smaller edema size and maximum edema diameter compared with patients with other NSCLC ( $\mathrm{P}=0.016$ and 0.010 , respectively), but not compared to those with adenocarcinoma ( $\mathrm{P}=0.065$ and 0.134 , respectively). By contrast, the tumor size reduction rate was significantly higher in patients with SCLC, compared to those with adenocarcinoma or other NSCLC $(\mathrm{P}=0.002$ and 0.005 , respectively). Furthermore, SCLC patients had a significantly lower PE-index compared to those with the other two histological types $(\mathrm{P}=0.001)$. Intracranial PFS and OS were not significantly different between SCLC and adenocarcinoma patients (Fig. 1A and B). However, intracranial PFS and OS differed significantly between adenocarcinoma and other NSCLC patients $(\mathrm{P}=0.018$ and 0.004 , respectively). Furthermore, intracranial PFS, unlike OS, was significantly different between SCLC and other NSCLC patients $(\mathrm{P}=0.048$ and 0.071 , respectively).

Effect of EGFR-TKIs on prognosis following WBRT. Out of the 44 adenocarcinoma patients, 19 were treated with EGFR-TKIs following WBRT. In 5 of these patients, EGFR mutations were confirmed (exon 21 point mutation, 1 patient; exon 19 deletion, 4 patients). EGFR-TKIs were administered as a second-line treatment in a further 14 patients who had never smoked, after they became resistant to standard platinum-based doublet chemotherapy. EGFR-TKIs were administered to treat locoregional disease in 6 patients, multiple pulmonary metastases in 7 patients, liver or bone metastases with/without locoregional disease in 3 patients and brain metastases in 3 patients. Distributions of the number of brain metastases and the RPA class were not significantly different between EGFR-TKI-treated and non-treated patients (Table III). According to the Kaplan-Meier survival curve, intracranial 
Table II. Clinical characteristics according to histological type.

\begin{tabular}{|c|c|c|c|c|}
\hline \multirow[b]{2}{*}{ Characteristics } & \multicolumn{3}{|c|}{ Histological type } & \multirow[b]{2}{*}{ P-value } \\
\hline & Adenocarcinoma & SCLC & Other NSCLC & \\
\hline Number of lesions & & & & 0.294 \\
\hline $1-3$ & $15(34 \%)$ & $7(46 \%)$ & $7(64 \%)$ & \\
\hline $4-6$ & $9(20 \%)$ & $4(27 \%)$ & $2(18 \%)$ & \\
\hline $7-10$ & $10(23 \%)$ & $0(0 \%)$ & $1(9 \%)$ & \\
\hline$\geq 11$ & $10(23 \%)$ & $4(27 \%)$ & $1(9 \%)$ & \\
\hline Total tumor size $\left(\mathrm{mm}^{2}\right)$ & & & & 0.650 \\
\hline Median (range) & $486(13-3635)$ & $355(40-1881)$ & $549(30-3348)$ & \\
\hline $\begin{array}{l}\text { Total edema size }\left(\mathrm{mm}^{2}\right) \\
\text { Median (range) }\end{array}$ & $1795(13-9894)$ & $593(40-4352)$ & 4598 (48-10744) & 0.041 \\
\hline $\begin{array}{l}\text { Tumor max diameter }(\mathrm{mm}) \\
\text { Median (range) }\end{array}$ & $19(2-53)$ & $20(6-37)$ & $25(6-62)$ & 0.424 \\
\hline $\begin{array}{l}\text { Edema max diameter }(\mathrm{mm}) \\
\text { Median (range) }\end{array}$ & $35(3-92)$ & $21(6-82)$ & $56(8-95)$ & 0.025 \\
\hline $\begin{array}{l}\text { PE-index } \\
\text { Median (range) }\end{array}$ & $3.07(1.00-20.33)$ & $1.30(1.00-9.80)$ & $5.29(1.49-19.47)$ & $<0.001$ \\
\hline Asymptomatic ratio & $45 \%$ & $60 \%$ & $18 \%$ & \\
\hline RPA class & & & & 0.295 \\
\hline I & $5(11 \%)$ & $3(20 \%)$ & $0(0 \%)$ & \\
\hline II & $29(66 \%)$ & $10(67 \%)$ & $6(55 \%)$ & \\
\hline III & $10(23 \%)$ & $2(13 \%)$ & $5(45 \%)$ & \\
\hline Tumor size reduction rate $(\mathrm{n}=50)$ & & & & $<0.001$ \\
\hline Median & $22.5 \%$ & $100 \%$ & $33.0 \%$ & \\
\hline Mean & $22.7 \%$ & $88.9 \%$ & $35.8 \%$ & \\
\hline Range & -121 to $100 \%$ & 52 to $100 \%$ & -19 to $95 \%$ & \\
\hline $\mathrm{CR}$ rate & $5.8 \%$ & $63.6 \%$ & $0 \%$ & \\
\hline MST (weeks) & 29.2 & 25.5 & 10.7 & \\
\hline
\end{tabular}

PE-index, ratio of tumor and peritumoral edema. SCLC, small-cell carcinoma; NSCLC, non-small-cell carcinoma; RPA, recursive partitioning analysis; CR, complete response; MST, median survival time.

PFS and OS were significantly higher in EGFR-TKI-treated patients $(\mathrm{P}=0.008$ and 0.004 , respectively; Fig. $2 \mathrm{~A}$ and $\mathrm{B})$. MST and progression-free median survival time were 18.8 (95\% CI, 9-28) and 8.9 (95\% CI, 7-18) weeks, respectively, in the non-treated group and 52.0 (95\% CI, 25-85) and 30.4 (95\% CI, 20-85) weeks, respectively, in the EGFR-TKItreated group.

\section{Discussion}

Although brain metastases from one primary tumor type may behave differently from those derived from another tumor type, the prognosis of patients with brain metastases is generally poor. Although the survival prognosis for lung cancer patients has improved due to the advances in systemic therapy, various cancers behave differently in terms of patterns of systemic disease and response to systemic therapy. These observations suggest that histological type may affect survival following WBRT due to different responses to recent systemic therapies (7-9). However, in a previous review by Gaspar et al it was reported that evidence supporting the effect of histopathology on WBRT treatment outcome was insufficient (17). Furthermore, with regards to survival benefit, the role of systemic treatment is controversial $(15,18)$ and WBRT remains the standard palliative treatment.

In this study, we investigated the survival of patients with lung cancer as defined by histological type following WBRT. Bergqvist et al previously reported a CR rate of $22 \%$ in SCLC, $5 \%$ in adenocarcinoma and $0 \%$ in squamous cell carcinoma patients (10) based on autopsy results, which is in accordance with the data presented in our study, although our value of the CR rate is slightly higher, due to imaging evaluation. The significant difference in survival following WBRT between adenocarcinoma and other NSCLC patients observed in the present study is in contrast to the results of a previous study by Kepka et al which demonstrated no significant difference 


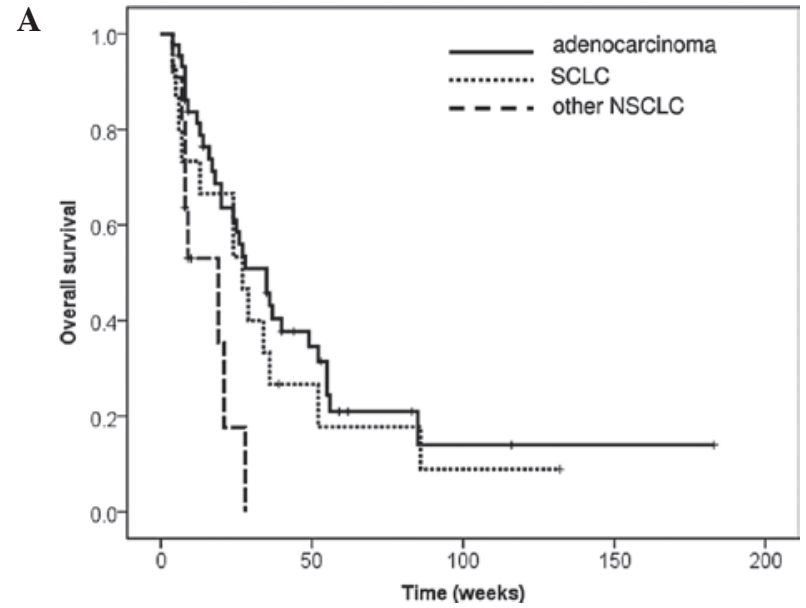

B

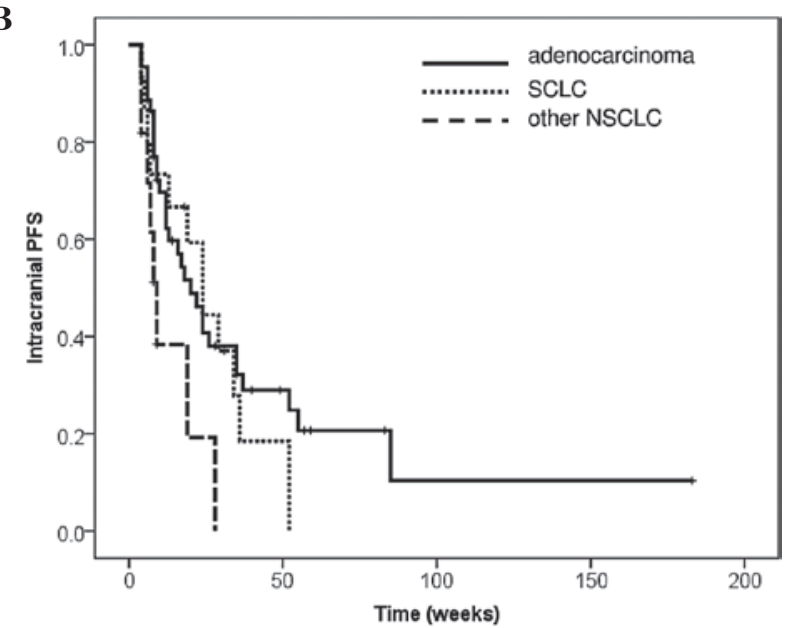

Figure 1. Kaplan-Meier survival curves showing (A) overall survival (OS) and (B) intracranial progression-free survival (PFS) according to histological type. Significant differences were observed between adenocarcinoma and other non-small-cell carcinoma (NSCLC) in intracranial PFS and OS $(\mathrm{P}=0.018$ and 0.004 , respectively). A significant difference was observed in intracranial PFS between small-cell lung carcinoma (SCLC) and other $\operatorname{NSCLC}(\mathrm{P}=0.048)$.

in survival following WBRT between NSCLC and SCLC, or adenocarcinoma and other NSCLC (11). Differences in the results between these two studies may be due to our use of EGFR-TKIs, which were not routinely used at the time of the study by Kepka et al (11). Results of the present study indicated that treatment with EGFR-TKIs leads to an improved prognosis and OS rate in all adenocarcinoma patients. In agreement with our findings, EGFR-TKIs have exhibited increased efficacy when used after WBRT. In addition, several studies reported the favorable effect of EGFR-TKIs on brain metastases $(15,19-22)$ and the improvement in the treatment response to EGFR-TKIs following radiotherapy (23-25).

Preclinical studies have also demonstrated the enhancing effect of previous irradiation on subsequent administration of EGFR-TKIs (26). Ceresoli et al conducted a prospective study to evaluate the efficacy of gefitinib in treating brain metastases (27). In their study on 41 patients treated with gefitinib, 18 patients who had previously been treated with WBRT exhibited improved disease control, compared to those who had not undergone WBRT (56 vs. 9\%). Ceresoli et al hypothesized that WBRT may increase the permeability of the blood-brain
Table III. Distribution of number of lesions and recursive partitioning analysis class (RPA).

\begin{tabular}{lccc}
\hline Variables & $\begin{array}{c}\text { Without } \\
\text { EGFR-TKIs }\end{array}$ & $\begin{array}{c}\text { With } \\
\text { EGFR-TKIs }\end{array}$ & P-value \\
\hline $\begin{array}{l}\text { No. of bone } \\
\text { metastases }\end{array}$ & & & 0.270 \\
$1-3$ & 10 & 5 & \\
$4-6$ & 6 & 3 & \\
$7-10$ & 6 & 4 & \\
$\geq 11$ & 3 & 7 & 0.596 \\
RPA class & 3 & 2 & \\
I & 15 & 14 & \\
II & 7 & 3 & \\
III & & & \\
\hline
\end{tabular}

EGFR, epidermal growth factor receptor; TKIs, tyrosine kinase inhibitors.

A

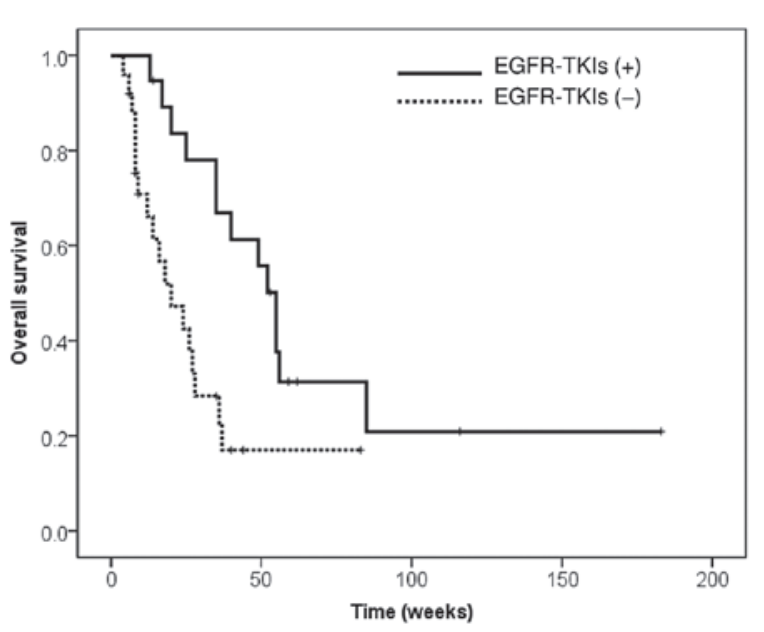

B

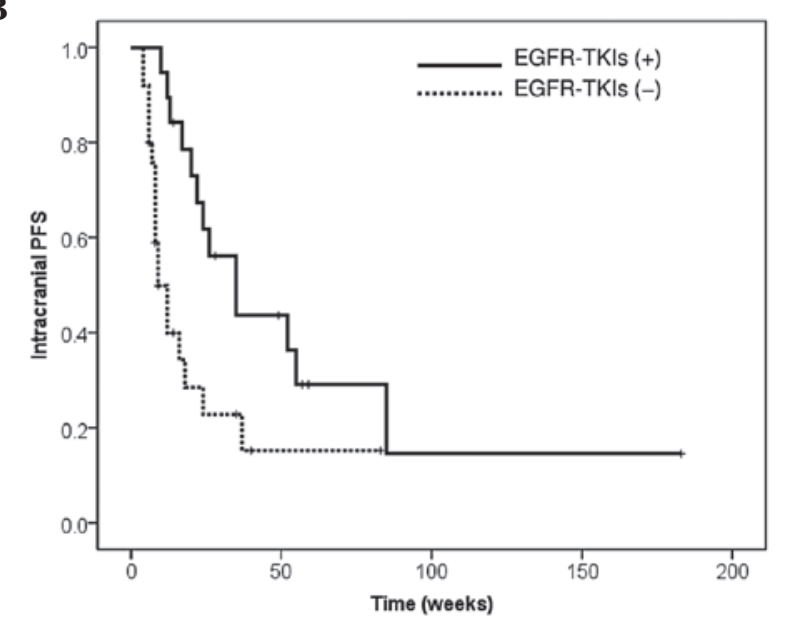

Figure 2. Kaplan-Meier survival curves showing (A) overall survival (OS) and (B) intracranial progression-free survival (PFS), stratified by the administration of epidermal growth factor receptor (EGFR) and tyrosine kinase inhibitors (TKIs), following whole-brain radiotherapy. Significant correlations between OS and the administration of EGFR-TKIs $(\mathrm{P}=0.004)$ and between intracranial PFS and the administration of EGFR-TKIs $(\mathrm{P}=0.008)$ were observed. 
barrier, permitting increased drug entry, or that the development of radiation-resistant clones induced by WBRT may be more sensitive to an alternative therapy. Another preclinical study demonstrated that irradiation activates the EGFR by releasing transforming growth factor $\alpha$ (28), whereas the effect of EGFR-TKIs on lung cancer was proven to be dependent on EGFR mutations $(29,30)$.

Differences in the effect of EGFR-TKIs on survival have been observed, depending on whether they were used as first- or second-line treatment and on the presence or absence of EGFR mutations. A previous study suggested that EGFR mutations should be evaluated to determine eligibility when EGFR-TKIs are used as first-line treatment (31). By contrast, other studies demonstrated that EGFR mutations are only associated with response and do not affect survival $(9,32)$. As a second-line treatment, EGFR-TKIs have been shown to have survival benefits. Since EGFR-TKIs were administered as a second-line treatment to several patients in the present retrospective study, EGFR mutations were not adequately assessed.

Furthermore, the results of the Southwest Oncology Group (SWOG) S0023 study, evaluating the efficacy of maintenance gefitinib following chemoradiation therapy, demonstrated inferior survival in patients receiving gefitinib as maintenance therapy (33). This inconsistency may be attributed to differences in patient characteristics, including race. However, the interaction between radiation and subsequent administration of EGFR-TKIs has not been fully elucidated $(34,35)$. Additional subgroup analysis considering these factors may provide useful insight on the subject.

WBRT aims to improve neurocognitive function and it has been reported that tumor size, peritumoral edema and tumor size reduction rate are related to neurocognitive function $(36,37)$. However, few studies have addressed this issue. In our study, pretreatment clinical characteristics were evaluated according to histological type. Peritumoral edema and asymptomatic ratio were less significant in patients with SCLC compared to those in patients with other histological tumor types. Peritumoral edema correlates strongly with neurological morbidity. Tabaka et al demonstrated, using specimens resected from patients who underwent surgery, that squamous cell carcinoma infiltrated into the surrounding brain tissue the most aggressively and that the border between brain metastasis and surrounding tissue was well defined in patients with SCLC (38). This histopathological feature may explain our finding of less extensive peritumoral edema in SCLC patients. Moreover, Wegner et al also reported a high asymptomatic ratio of up to $73 \%$ in a study on stereotactic radiosurgery for patients with brain metastases from SCLC (39).

Taken together, these findings indicate that early detection of brain metastases in patients with SCLC may require regular brain imaging examinations, regardless of the presence or absence of symptoms. Early detection of intracranial recurrence by regular imaging examination and subsequent reirradiation may become one of the strategies in following up patients with SCLC. Due to the poor response to radiotherapy and the limited number of metastases, more aggressive approaches, such as surgery or stereotactic radiosurgery, may be suitable for patients with other NSCLC in the case of recurrence. However, our results must be interpreted with caution, considering the selection bias due to the retrospective nature of this study, race differences and our relatively limited sample size. Thus, additional investigations are required to validate the benefits of such treatment strategies.

In conclusion, in this study we presented the clinical characteristics associated with different histological types of lung cancer, which may provide useful information for the management of brain metastases from lung cancer. Patients with SCLC exhibited a lower degree of peritumoral edema, whereas patients with adenocarcinoma, particularly those treated with EGFR-TKIs, exhibited improved survival rates.

\section{References}

1. Coia LR: The role of radiation therapy in the treatment of brain metastases. Int J Radiat Oncol Biol Phys 23: 229-238, 1992.

2. Sperduto PW, Chao ST, Sneed PK, et al: Diagnosis-specific prognostic factors, indexes, and treatment outcomes for patients with newly diagnosed brain metastases: a multi-institutional analysis of 4,259 patients. Int J Radiat Oncol Biol Phys 77: 655-661, 2009.

3 Tsao MN, Rades D, Wirth A, et al: Radiotherapeutic and surgical management for newly diagnosed brain metastasis(es): An American Society for Radiation Oncology evidence-based guideline. Pract Radiat Oncol 2: 210-225, 2012.

4. Lagerwaard FJ, Levendag PC, Nowak PJ, Eijkenboom WM, Hanssens PE and Schmitz PI: Identification of prognostic factors in patients with brain metastases: a review of 1,292 patients. Int J Radiat Oncol Biol Phys 43: 795-803, 1999.

5. Newman SJ and Hansen HH: Proceedings: Frequency, diagnosis, and treatment of brain metastases in 247 consecutive patients with bronchogenic carcinoma. Cancer 33: 492-496, 1974.

6. Sen M, Demiral AS, Cetingöz R, Alanyali H, Akman F, Sentürk D and Kinay M: Prognostic factors in lung cancer with brain metastasis. Radiother Oncol 46: 33-38, 1998.

7. Scagliotti GV, Parikh P, von Pawel J, et al: Phase III study comparing cisplatin plus gemcitabine with cisplatin plus pemetrexed in chemotherapy-naive patients with advanced-stage non-small-cell lung cancer. J Clin Oncol 26: 3543-3551, 2008.

8. Mok TS, Wu YL, Thongprasert S, et al: Gefitinib or carboplatin-paclitaxel in pulmonary adenocarcinoma. N Engl J Med 361: 947-957, 2009.

9. Tsao MS, Sakurada A, Cutz JC, et al: Erlotinib in lung cancermolecular and clinical predictors of outcome. N Engl J Med 353: 133-144, 2005.

10. Bergqvist M, Brattström D, Bennmarker H, Wagenius G, Riska $H$ and Brodin O: Irradiation of brain metastases from lung cancer: a retrospective study. Lung Cancer 20: 57-63, 1998.

11. Kepka L, Cieslak E, Bujko K, Fijuth J and Wierzchowski M: Results of the whole-brain radiotherapy for patients with brain metastases from lung cancer: the RTOG RPA intra-classes analysis. Acta Oncol 44: 389-398, 2005.

12. Ryan GF, Ball DL and Smith JG: Treatment of brain metastases from primary lung cancer. Int J Radiat Oncol Biol Phys 31: 273-278, 1995.

13. Penel N, Brichet A, Prevost B, Duhamel A, Assaker R, Dubois F and Lafitte JJ: Pronostic factors of synchronous brain metastases from lung cancer. Lung Cancer 33: 143-154, 2001.

14. Sánchez de Cos J, Sojo González MA, Montero MV, Pérez Calvo MC, Vicente MJ and Valle MH: Non-small cell lung cancer and silent brain metastasis. Survival and prognostic factors. Lung Cancer 63: 140-145, 2009.

15. Yamanaka R: Medical management of brain metastases from lung cancer (Review). Oncol Rep 22: 1269-1276, 2009.

16. Gaspar LE, Scott C, Rotman M, Asbell S, Phillips T, Wasserman T, McKenna WG and Byhardt R: Recursive partitioning analysis (RPA) of prognostic factors in three Radiation Therapy Oncology Group (RTOG) brain metastases trials. Int J Radiat Oncol Biol Phys 37: 745-751, 1997.

17. Gaspar LE, Mehta MP, Patchell RA, et al: The role of whole brain radiation therapy in the management of newly diagnosed brain metastases: a systematic review and evidence-based clinical practice guideline. J Neurooncol 96: 17-32, 2010.

18. Langer CJ and Mehta MP: Current management of brain metastases, with a focus on systemic options. J Clin Oncol 23: 6207-6219, 2005 
19. Cappuzzo F, Ardizzoni A, Soto-Parra H, Gridelli C, Maione P, Tiseo M, Calandri C, Bartolini S, Santoro A and Crinò L: Epidermal growth factor receptor targeted therapy by ZD 1839 (Iressa) in patients with brain metastases from non-small cell lung cancer (NSCLC). Lung Cancer 41: 227-231, 2003.

20. Poon AN, Ho SS, Yeo W and Mok TS: Brain metastasis responding to gefitinib alone. Oncology 67: 174-178, 2004.

21. Popat S, Hughes S, Papadopoulos P, Wilkins A, Moore S, Priest K, Meehan L, Norton A and O'Brien M: Recurrent responses to non-small cell lung cancer brain metastases with erlotinib. Lung Cancer 56: 135-137, 2007.

22. Gounant V, Wislez M, Poulot V, Khalil A, Lavole A, Cadranel J and Milleron B: Subsequent brain metastasis responses to epidermal growth factor receptor tyrosine kinase inhibitors in a patient with non-small-cell lung cancer. Lung Cancer 58 425-428, 2007.

23. Shukuya T, Takahashi T, Naito T, et al: Continuous EGFR-TKI administration following radiotherapy for non-small cell lung cancer patients with isolated CNS failure. Lung Cancer 74: 457-461, 2011

24. Namba Y, Kijima T, Yokota S, et al: Gefitinib in patients with brain metastases from non-small-cell lung cancer: review of 15 clinical cases. Clin Lung Cancer 6: 123-128, 2004.

25. Hotta K, Kiura K, Ueoka H, Tabata M, Fujiwara K, Kozuki T, Okada T, Hisamoto A and Tanimoto M: Effect of gefitinib ('Iressa', ZD1839) on brain metastases in patients with advanced non-small-cell lung cancer. Lung Cancer 46: 255-261, 2004.

26. Bianco C, Tortora G, Bianco R, et al: Enhancement of antitumor activity of ionizing radiation by combined treatment with the selective epidermal growth factor receptor-tyrosine kinase inhibitor ZD1839 (Iressa). Clin Cancer Res 8: 3250-3258, 2002.

27. Ceresoli GL, Cappuzzo F, Gregorc V, Bartolini S, Crinò L and Villa E: Gefitinib in patients with brain metastases from non-small-cell lung cancer: a prospective trial. Ann Oncol 15: 1042-1047, 2004

28. Dent P, Reardon DB, Park JS, Bowers G, Logsdon C, Valerie K and Schmidt-Ullrich R: Radiation-induced release of transforming growth factor alpha activates the epidermal growth factor receptor and mitogen-activated protein kinase pathway in carcinoma cells, leading to increased proliferation and protection from radiationinduced cell death. Mol Biol Cell 10: 2493-2506, 1999.
29. Patez JG, Janne PA, Lee JC, et al: EGFR mutations in lung cancer: correlation with clinical response to gefitinib therapy. Science 304: 1497-1500, 2004

30. Lynch TJ, Bell DW, Sordella R, et al: Activating mutations in the epidermal growth factor receptor underlying responsiveness of non-small-cell lung cancer to gefitinib. N Engl J Med 350: 2129-2139, 2004

31. Salgia R, Hensing T, Campbell N, Salama AK, Maitland M, Hoffman P, Villaflor V and Vokes EE: Personalized treatment of lung cancer. Semin Oncol 38: 274-283, 2011.

32. Cappuzzo F, Hirsch FR, Rossi E, et al: Epidermal growth factor receptor gene and protein and gefitinib sensitivity in non-smallcell lung cancer. J Natl Cancer Inst 97: 643-655, 2005.

33. Kelly K, Chansky K, Gaspar LE, Albain KS, Jett J, Ung YC, Lau DH, Crowley JJ and Gandara DR: Phase III trial of maintenance gefitinib or placebo after concurrent chemoradiotherapy and docetaxel consolidation in inoperable stage III non-small-cell lung cancer: SWOG S0023. J Clin Oncol 26: 2450-2456, 2008.

34. Provencio M, Sánchez A, Garrido P and Valcárcel F: New molecular targeted therapies integrated with radiation therapy in lung cancer. Clin Lung Cancer 11: 91-97, 2010.

35. Xu Y, Zhang Y and Ma S: EGFR inhibitors with concurrent thoracic radiation therapy for locally advanced non-small cell lung cancer. Lung Cancer 73: 249-255, 2011.

36. Aoyama H, Tago M, Kato N, et al: Neurocognitive function of patients with brain metastasis who received either whole brain radiotherapy plus stereotactic radiosurgery or radiosurgery alone. Int J Radiat Oncol Biol Phys 68: 1388-1395, 2007.

37. Meyers CA, Smith JA, Bezjak A, et al: Neurocognitive function and progression in patients with brain metastases treated with whole-brain radiation and motexafin gadolinium: results of a randomized phase III trial. J Clin Oncol 22: 157-165, 2004.

38. Tabaka J, Nowacki P and Pankowski J: The interaction between lung cancer metastases to the brain and their surroundings. Folia Neuropathol 44: 42-49, 2006.

39. Wegner RE, Olson AC, Kondziolka D, Niranjan A, Lundsford LD and Flickinger JC: Stereotactic radiosurgery for patients with brain metastases from small cell lung cancer. Int J Radiat Oncol Biol Phys 81: e21-e27, 2011. 